\title{
An extended weight model for GPS phase observations
}

\author{
Andreas Wieser and Fritz K. Brunner \\ Engineering Surveying and Metrology, Graz University of Technology, A-8010 Graz, Austria
}

(Received December 20, 1999; Revised July 7, 2000; Accepted July 9, 2000)

\begin{abstract}
Low elevation data may be included in the processing of small scale engineering GPS applications, in order to improve satellite geometry and reduce the required observation time. Careful weighting of the phase observations is needed then, because of increased noise and systematic errors. The measured carrier-to-noise-power-density ratio $(\mathrm{C} / \mathrm{N} 0)$ has proven to be an excellent tool for the estimation of the random errors of the phase observables. In this paper an extended weight model using robust estimation is presented, in particular the Danish Method. This model combines the information inherent in $\mathrm{C} / \mathrm{N} 0$ and the residuals of the double differenced phase observations in order to model random errors and signal distortion effects.
\end{abstract}

\section{Introduction}

The accuracy of GPS based point determination in small scale engineering applications is mainly limited by multipath and signal diffraction. Often the observation sites are determined by positional requirements rather than by ideal observation conditions which increases the probability of obstructions and signal distortions. In order to improve satellite geometry and reduce the required observation time, low elevation data is included in the processing but this increases noise and systematic errors. Thus careful weighting of the phase observations is needed.

The carrier-to-noise-power-density ratio (C/N0), i.e. the ratio of the power level of the signal carrier to the noise power in a $1 \mathrm{~Hz}$ bandwidth (Langley, 1997), is a normalized measure of the signal-to-noise value. The $\mathrm{C} / \mathrm{N} 0$ has proven to be an excellent tool for the estimation of the random errors of the phase observables. Due to the strong correlation between the satellite elevation and the $\mathrm{C} / \mathrm{N} 0$, a template function can be determined to derive the expected $\mathrm{C} / \mathrm{N} 0$ for an elevation angle and the specific receiver-antenna combination. The difference $(\Delta)$ between the C/NO observation and the template value is an indicator for systematic signal distortion (Brunner et al., 1999) which is also shown a posteriori by the residuals of the phase data.

In this paper an extended weight model is presented which allows to combine the information inherent in $\mathrm{C} / \mathrm{N} 0$ and residuals in order to take random errors and diffraction effects into account. Using this model, the precision of the solution is improved and consequently the required observation time can be reduced. The model presented is a further step towards a more general weight model that uses measured and estimated quality parameters of the observations.

Copy right $(\mathrm{c}$ The Society of Geomagnetism and Earth, Planetary and Space Sciences (SGEPSS); The Seismological Society of Japan; The Volcanological Society of Japan; The Geodetic Society of Japan; The Japanese Society for Planetary Sciences.

\section{C/NO and SIGMA Weight Models}

\subsection{SIGMA- $\epsilon$ weight model}

The standard deviation of the phase observations in the phase lock loop of a GPS receiver is a function of the C/N0, the bandwidth and the carrier frequency (Langley, 1997). The C/N0 is usually determined by a voltage measurement in the receiver and is stored in the binary observation file. It is an independent measure of the quality of the phase observations, accessible at processing time.

The SIGMA- $\epsilon$ weight model (Hartinger and Brunner, 1999) links the $\mathrm{C} / \mathrm{N} 0$ observable to the variance of the phase observation by means of the model parameter $C_{i}$. We have found that some receiver-antenna combinations require an additional additive term, $V_{i}$. The SIGMA- $\epsilon$ model now reads:

$$
\sigma_{\phi}^{2}=V_{i}+C_{i} \cdot 10^{-\frac{\mathrm{C} / \mathrm{N} 0}{10}}
$$

$$
\begin{aligned}
& \sigma_{\phi}^{2} \quad \ldots \quad \text { variance of undifferenced } \\
& \begin{array}{lll} 
& & \text { phase observation } \\
V_{i}, C_{i} & \ldots & \text { model parameters }
\end{array} \\
& {\left[\mathrm{m}^{2}\right],\left[\mathrm{m}^{2} \mathrm{~Hz}\right]} \\
& i \quad \text {.. receiver/antenna type } \\
& \text { C/NO } \quad . \quad \text { measured carrier-to-noise- } \\
& \text { power-density ratio }[\mathrm{dB}-\mathrm{Hz}]
\end{aligned}
$$

Figure 1 shows a time series of actual standard deviation of double differenced phase observations (DD) and SIGMA- $\epsilon$ output after calibration of the parameters. The data (Rogue SNR8000/DORNE MARGOLIN B) of the figure were provided by Gunnar Elgered, Onsala. The constants of the SIGMA- $\epsilon$ model were estimated as $C_{\mathrm{L} 1}=0.244 \mathrm{~m}^{2} \mathrm{~Hz}$, $V_{\mathrm{L} 1}=0, C_{\mathrm{L} 2}=0.77 \cdot 10^{-3} \mathrm{~m}^{2} \mathrm{~Hz}$ and $V_{\mathrm{L} 2}=0.88 \cdot 10^{-6} \mathrm{~m}^{2}$. Although the SIGMA- $\epsilon$ output is mainly elevation dependent (ref. Subsection 2.2), it is superior to a simple function of elevation since it reflects the actual signal quality. 


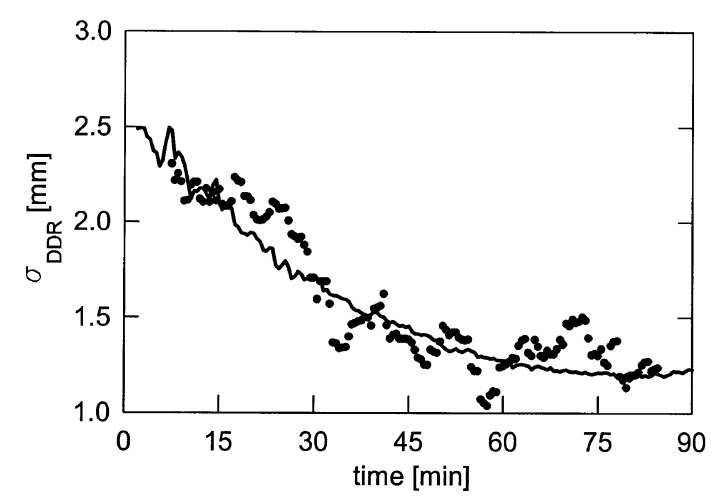

Fig. 1. Random measurement noise $\sigma_{\mathrm{DDR}}$ (dots) and its estimation by SIGMA- $\epsilon$ (line) for L1 double difference PRN13-02. $\sigma_{\text {DDR }}$ calculated from $\mathrm{DD}$ residuals with a sliding window of 30 epochs.

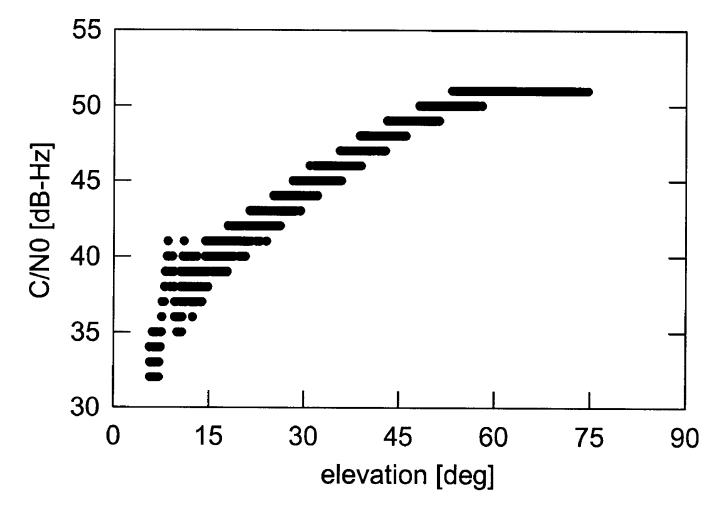

Fig. 2. C/N0 observations (L1, Leica SR399, external antenna without ground plane. Session: $1 \mathrm{~h}, 3$ s sampling, all satellites in view).

\subsection{SIGMA- $\Delta$ weight model}

As can be seen from Fig. 2, the C/N0 is mainly elevation dependent. Therefore it is possible to model the $\mathrm{C} / \mathrm{N} 0$ expected for a satellite at a certain elevation by a template function. Experiments have shown that signal distortion coincides with a deviation of the actual C/N0 from the template value (Brunner et al., 1999). We express this additional signal attenuation via $\Delta$ (see Fig. 3 ).

Signal distortion may now be treated in the adjustment by reducing the weights of observations when $\Delta \neq 0$. The empirical SIGMA- $\Delta$ weight model achieves this by increasing the a priori variances of the observations with an increased $|\Delta|$ :

$$
\sigma_{\phi}^{2}=V_{i}+C_{i} \cdot 10^{-\frac{\mathrm{C} / \mathrm{N} 0-\alpha \cdot|\Delta|}{10}}
$$

$$
\begin{aligned}
& \Delta \quad \cdots \quad \text { deviation from } \mathrm{C} / \mathrm{N} 0 \\
& \text { template }[\mathrm{dB}-\mathrm{Hz}] \\
& \alpha \quad \ldots \quad \text { empirical constant factor (2.0) }
\end{aligned}
$$

$|\Delta|$ takes into account that signal distortion may also increase the signal-to-noise ratio (Allnutt, 1989). This is a minor modification to (Brunner et al., 1999) where $\Delta$ was used instead of $|\Delta|$.

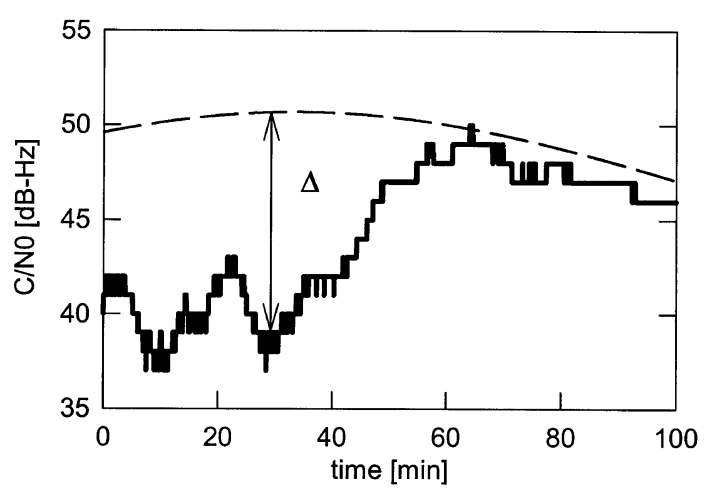

Fig. 3. C/N0 observations of PRN01 (solid), template function (dashed) and definition of $\Delta$ during time of signal distortion (3 s sampling, L1, Leica SR399, external antenna).

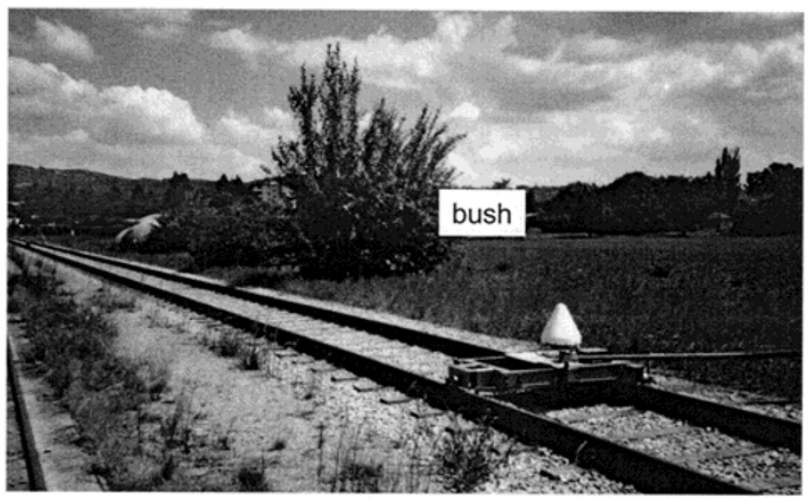

Fig. 4. Survey trolley on railway track, close to bush.

\subsection{Example: Kinematic experiment}

When processing GPS phase observations, we apply the SIGMA- $\epsilon$ model routinely as basic weight model. In addition, the SIGMA- $\Delta$ model has been successfully used, for example with the following kinematic railway track survey (Brunner et al., 1999).

A straight part of a railway track in Austria (length approx. $150 \mathrm{~m}$ ) was measured with a Leica SR399 receiver and a choke-ring antenna mounted on a survey trolley. GPS phase observations were recorded with an observation rate of $1 \mathrm{~s}$ and an elevation cut-off of $10^{\circ}$ while the trolley was moved along the track. The reference station was set up close to the track (kinematic baseline length $<100 \mathrm{~m}$ ).

At a certain along track position of the trolley (approx. $95 \mathrm{~m}$ ), a nearby bush imposed strong diffraction on the signals of satellite PRN03, see Figs. 4 and 5. The diffraction is clearly indicated by the $\mathrm{C} / \mathrm{N} 0$ values, which drop to $36 \mathrm{~dB}$ $\mathrm{Hz}$, well below the template value of $48 \mathrm{~dB}-\mathrm{Hz}$.

A detailled discussion of the data is given in Brunner et al. (1999). As can be seen from the time series of the cross track error in Fig. 6, the use of the SIGMA models clearly improves the results of kinematic processing. The bias still visible at positions close to $95 \mathrm{~m}$ - indicates that the C/N0 values may not always provide sufficient information about the amount of signal distortion. The following section highlights the main reasons for the limited applicability and 


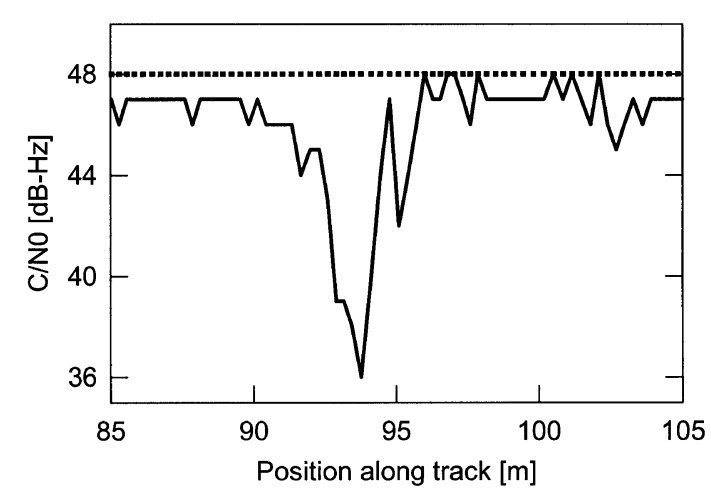

Fig. 5. C/N0 observation (solid) and template (dotted) of PRN03 showing strong diffraction effect at position $94 \mathrm{~m}$ (Leica SR399, external antenna).

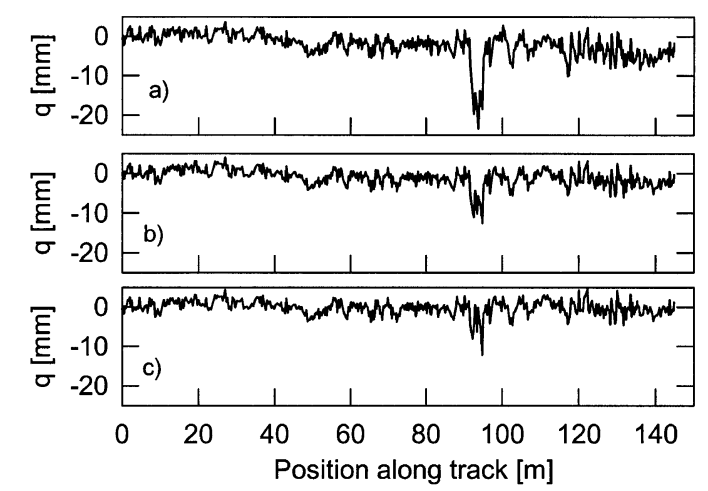

Fig. 6. Time series of cross track deviation $q$ of estimated trajectory. Results using (a) equal weights, (b) SIGMA- $\epsilon$, (c) SIGMA- $\Delta$.

demonstrates the necessity for an extension of the models.

\section{Limitation of SIGMA Models}

The SIGMA models rely on the C/N0 as a measure of signal quality and signal distortion. Unfortunately, there is no straightforward functional relationship between $\mathrm{C} / \mathrm{N} 0$ attenuation and phase error in real world situations. Axelrad et al. (1994) have presented a method to model the phase error as a function of the signal-to-noise ratio (SNR), based on spectral analysis of the SNR and manual identification of multipath constituents. Although the method seems promising, it is very difficult to be implemented for non-interactive processing, and it is likely to fail in complex (real world) environments. Furthermore, it is not applicable to estimate the phase error with signal diffraction, since this is not indicated by SNR oscillations.

The limits of $\mathrm{C} / \mathrm{N} 0$ as a direct measure of a phase error are discussed for the two main signal distortion scenarios diffraction and multipath.

\subsection{Diffraction}

Figure 7 shows a typical diffraction situation for a short baseline $(100 \mathrm{~m})$, reported by Brunner et al. (1999). The rover station is close to a narrow building, which totally obstructs the direct signal from PRN01 during the first $30 \mathrm{~min}$ of the session. The double difference phase residuals (DDR) may increase to a theoretically unbound value, depending

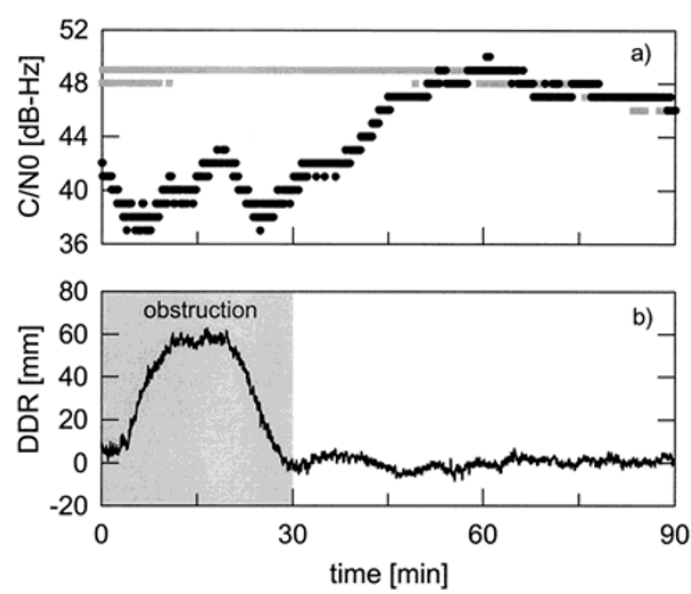

Fig. 7. (a) $\mathrm{C} / \mathrm{N} 0$ of PRN01 at reference (grey squares) and rover station (dots), (b) L1 double difference residuals PRN01-15. No direct signal of PRN01 during the first $0.5 \mathrm{~h}$ at rover station. $3 \mathrm{~s}$ sampling.

only on the additional path length of the affected signal while the receiver tracks the satellite. The $\mathrm{C} / \mathrm{N} 0$ is considerably lower for the affected signal, but does not drop below the acquisition threshold and so PRN01 is continuously tracked while it passes behind the obstacle.

Intermediately the $\mathrm{C} / \mathrm{N} 0$ recovers while the satellite is still not geometrically visible. This may be due to obstacle gain, as mentioned by Allnutt (1989, pp. 319-328). The DDR do not show a simultaneous improvement.

The residuals decrease to $\mathrm{mm}$ level as the satellite approaches the end of the obstructed period. The C/N0 only recovers half an hour later, and thus still indicates strong distortion. Actually, there is only a small multipath effect left, as can be seen from the typical low frequency oscillations of the DDR until minute 60 .

We conclude that weighting based exclusively on the C/N0 and its attenuation $\Delta$ fails under certain circumstances, since it

- introduces too high weights for observations under diffraction if obstacle gain occurs - thus creating a biased solution - , and

- introduces too small weights for the observations when the direct signal path is close to the border of an obstruction - thus unnecessarily weakening the geometry.

\subsection{Multipath}

The typical pattern of DDR affected by multipath is an oscillation with approximately zero mean and a maximum amplitude of $\lambda / 4$, i.e. $4.8 \mathrm{~cm}$ for L1 (Eissfeller, 1997). Corresponding oscillations about the template value can also be identified for the C/N0, see Fig. 8. The time lag between the two oscillations is a function of all multipath constituents and can hardly be modelled if there is an unknown number of reflecting objects.

We conclude that in the presence of multipath

- $\Delta=0$ does not necessarily identify the epochs of zero phase error - quite on the contrary it may coincide with the epochs of maximum phase error, and 

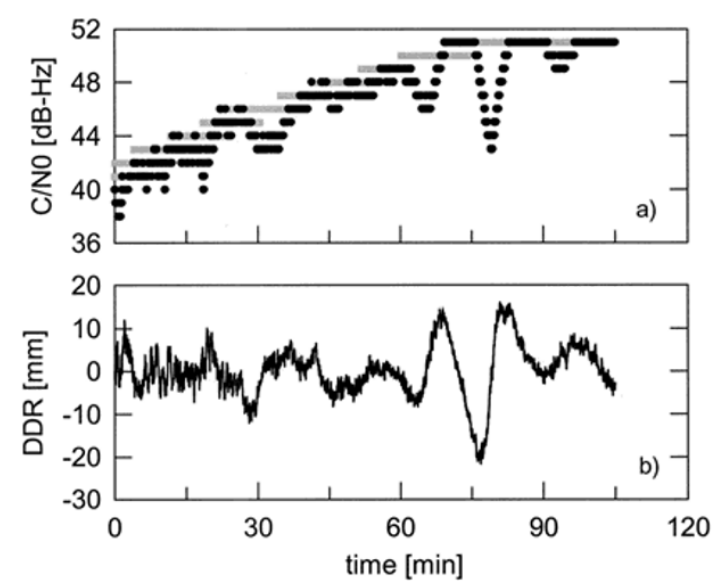

Fig. 8. (a) $\mathrm{C} / \mathrm{N} 0$ of PRN29 at reference (grey squares) and rover station (dots), (b) L1 double difference residuals PRN29-15. 3 s sampling.

- a local maximum of $|\Delta|$ does not always coincide with a maximum phase error - rather it may coincide with a very small error.

\section{Robust Estimation}

Robust estimation techniques reduce the influence of outliers on the result. The distorted signals of the cases shown above, are not really outliers but biased observations. Nevertheless, if the bias cannot or shall not be modelled, these observations must be assigned low weights to prevent a deteriorating effect on the results.

We have implemented the Danish Method (Krarup et al., 1980) to investigate the potential of the residuals as an input to an extended weight model for coping with mutlipath and diffraction. The influence of observations not consistent with the majority of observations is limited in the Danish Method. The estimation procedure is realized by an iterative least squares adjustment with modification of the weights of observations with large normalized residuals.

Our implementation is based on a standard Gauss-Markov model (3) of double differenced phase observations, and the initial cofactor matrix $\mathbf{Q}_{\mathbf{y y}}$ of the observations is calculated by the SIGMA- $\epsilon$ model:

$$
\begin{gathered}
\mathbf{y}=\mathbf{A} \cdot \xi-\mathbf{e} \\
\mathbf{e} \sim\left(\mathbf{0}, \sigma_{0}^{2} \mathbf{Q} \mathbf{y y}\right)
\end{gathered}
$$

$\begin{array}{lll}\mathbf{y} & \ldots & \text { vector of observations } \\ \mathbf{A} & \ldots & \text { Jacobian matrix } \\ \xi & \ldots & \text { vector of parameters } \\ \mathbf{e} & \ldots & \text { vector of residuals } \\ \sigma_{0}^{2} & \ldots & \text { a priori variance factor } \\ \mathbf{Q y y} & \ldots & \begin{array}{l}\text { cofactor matrix } \\ \text { of observations }\end{array}\end{array}$

After the inversion of the normal equation system, the residuals $\hat{\mathbf{e}}$ are estimated. Furthermore, the standard deviation of the residuals is estimated using a fixed, known a priori variance factor $\sigma_{0}^{2}$.

$$
\hat{\mathbf{e}}=\left(\mathbf{A}\left(\mathbf{A}^{\mathrm{T}} \mathbf{Q}_{\mathbf{y} \mathbf{y}}^{-1} \mathbf{A}\right)^{-1} \mathbf{A}^{\mathrm{T}} \mathbf{Q}_{\mathbf{y} \mathbf{y}}^{-1}-\mathbf{I}\right) \cdot \mathbf{y}
$$

$$
\begin{gathered}
\hat{e}_{i}=\hat{\mathbf{e}}(i) \\
\mathbf{Q}_{\hat{\mathbf{e}} \hat{\mathbf{e}}}=\mathbf{Q}_{\mathbf{y y}}-\mathbf{A}\left(\mathbf{A}^{\mathrm{T}} \mathbf{Q}_{\mathbf{y y}}^{-1} \mathbf{A}\right)^{-1} \mathbf{A}^{\mathrm{T}} \\
\sigma_{\hat{e}_{i}}=\sigma_{0} \cdot \sqrt{\mathbf{Q}_{\hat{\mathbf{e}} \hat{\mathbf{e}}}(i, i)}
\end{gathered}
$$

The normalized residuals $\tilde{e}_{i}$ are then calculated using the estimated residuals and their standard deviation $\sigma_{\hat{e}_{i}}$.

$$
\tilde{e}_{i}=\frac{\hat{e}_{i}}{\sigma_{\hat{e}_{i}}}
$$

According to the Danish Method, we compare these normalized residuals $\tilde{e}_{i}$ to a predetermined threshold $c$, e.g. $c=3$ (Jørgensen, 1985). Alternatively, it is possible to calculate an individual $c_{i}$ for each observation, corresponding to a chosen probability of $\tilde{e}_{i}$ being larger than $c_{i}(\mathrm{Xu}, 1993)$. With uncorrelated observations $\tilde{e}_{i}$ is also the test statistic for outlier detection by data snooping (Baarda, 1968).

For each observation a weight factor $f_{i}$ is calculated:

$$
f_{i}=\left\{\begin{array}{cl}
\exp \left(-\frac{\left|\tilde{e}_{i}\right|}{c}\right) & \text { if }\left|\tilde{e}_{i}\right|>c \\
1 & \text { else }
\end{array}\right.
$$

Finally, the weight matrix $\mathbf{Q}_{\mathbf{y} \mathbf{y},(k+1)}^{-1}$ for the next iteration is determined using the initial cofactor matrix $\mathbf{Q}_{\mathbf{y y} \mathbf{y},(1)}^{-1}$ and the vector $\mathbf{f}_{(k)}$ of weight factors from the $k$-th iteration. This may formally be expressed as a functional relation $G$ :

$$
\mathbf{Q}_{\mathbf{y y},(k+1)}^{-1}=G\left(\mathbf{Q}_{\mathbf{y y},(1)}^{-1}, \mathbf{f}_{(k)}\right)
$$

Actually the diagonal elements of the $\mathbf{Q}_{\mathbf{y y}}$,(1) matrix are divided by the corresponding weight factors, thus changing the individual weights of the double difference observations. This simple procedure is based on the following conditions:

1) Inter-epoch correlations are neglected.

2) One station is chosen as reference station for all single differences.

3) One satellite per epoch is chosen as reference satellite for all double differences of this epoch.

4) Within each epoch only $m<n$ observations are marked as outliers, where $n$ is the total number of observations of the same baseline and frequency within this epoch. In this case the outliers actually occurred in the roverstation to rover-satellite observations.

If necessary, one can usually obtain the situation of condition 4 by changing the reference satellite of the epoch. Otherwise, it is possible to modify the variances of the undifferenced observations and calculate $\mathbf{Q y y}_{\mathbf{y},(k+1)}$ by variance propagation.

Selecting a different reference station or reference satellite for certain observations does not change the final result. However, the required modifications to $\mathbf{Q}_{\mathbf{y y}}$,(1) would become more involved.

A new iteration of the adjustment is performed with the updated $\mathbf{Q}_{\mathbf{y y}}$ matrix. The iterations stop if $\mathbf{f}_{(k+1)}=\mathbf{f}_{(k)}$ within a preset limit. Then, the final result and the final 
weights are obtained. Note that corresponding to the Danish Method, the normalization factor of the residuals remains constant for all iterations. Therefore, $\sigma_{\hat{e}_{i}}$ is only calculated during the first iteration and used in Eq. (9) for all subsequent iterations.

\section{Results}

\subsection{Diffraction}

The data presented in Fig. 7 was reprocessed with the combination of SIGMA- $\epsilon$ and the Danish Method. The a priori standard deviations $\sigma_{y}$ of the individual double difference observations are used to compose the $\mathbf{Q}_{\mathbf{y y}}$ matrix. Figure 9 (bottom) shows the time series of $\sigma_{y}$ of a certain double difference, according to the SIGMA- $\epsilon$ model and the combined model (SIGMA- $\epsilon$ plus Danish Method). We see that the Danish Method successfully identifies the large phase errors at minutes 10-25 and yields high a priori standard deviation $\sigma_{y}$ for the corresponding observations.

Figure 10 (top) shows how the bias in the PRN01-15 double difference, due to diffraction of the PRN01 signal at the rover station, is absorbed by several double difference observations for an epoch-to-epoch processing using the SIGMA-
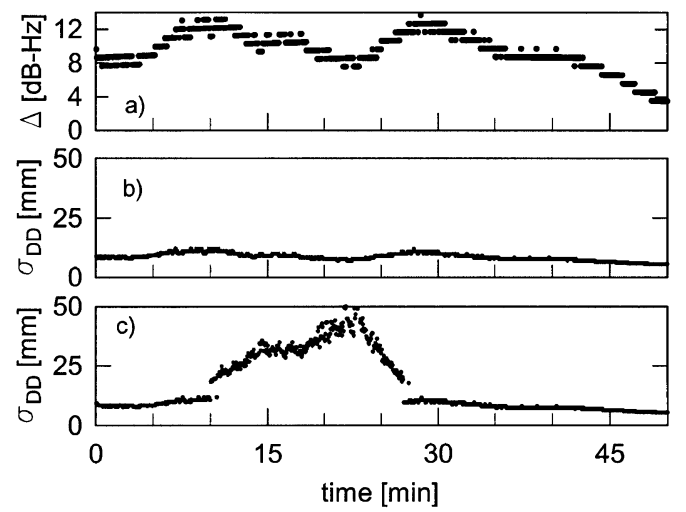

Fig. 9. $\Delta$ of PRN01 at rover station (a). $\sigma_{\mathrm{DDR}}=\sigma_{y}$ of PRN01-15: SIGMA- $\epsilon$ (b) and SIGMA- $\epsilon$ plus Danish Method (c). 3 s sampling.
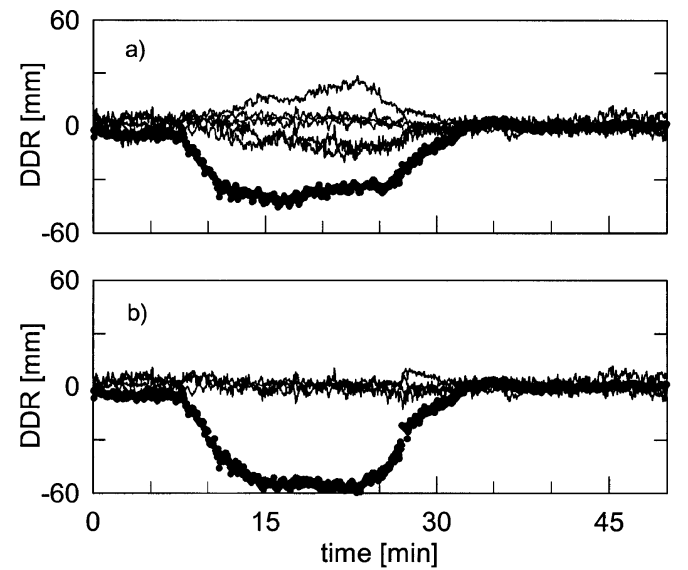

Fig. 10. Time series of all L1 double difference residuals: (a) SIGMA- $\epsilon$, (b) SIGMA- $\epsilon$ plus Danish Method. PRN01-15 (dots), other satellite pairs (solid). $\epsilon$ model. The additional application of the Danish Method clearly identifies the bad observations (bottom) and thus the bias only shows up in the residuals of these observations.

The time series of the coordinates affirms this improvement, see Fig. 11. The bias of the dY component — about $3 \mathrm{~cm}$ with SIGMA- $\epsilon$ - is almost completely removed. Also the results for the $\mathrm{dZ}$ component are improved significantly.

At the first and last epoch where the Danish Method identifies an outlier, a sudden change in the a priori sigma of the bad double difference observations causes a jump in the time series of the results. This is due to the weight factor $f_{j}$ which jumps from 1 to $1 / e$ when the normalized residuals pass the threshold value, s. Eq. (10). A more sophisticated weight model, that combines the C/N0 and DDR information would be able to introduce smooth modifications to the initial weights, if the bias changes smoothly.

\subsection{Multipath}

Figure 12 shows the normalized residuals of PRN15-29 of a 10 min subsession of static data (center). The corresponding C/N0 attenuation of PRN29 at the rover site is plotted in the top row. The pattern of the residuals indicates multipath. Note the time lag between $\mathrm{C} / \mathrm{N} 0$ and residuals of about a quarter of the multipath period. Due to the geometry the

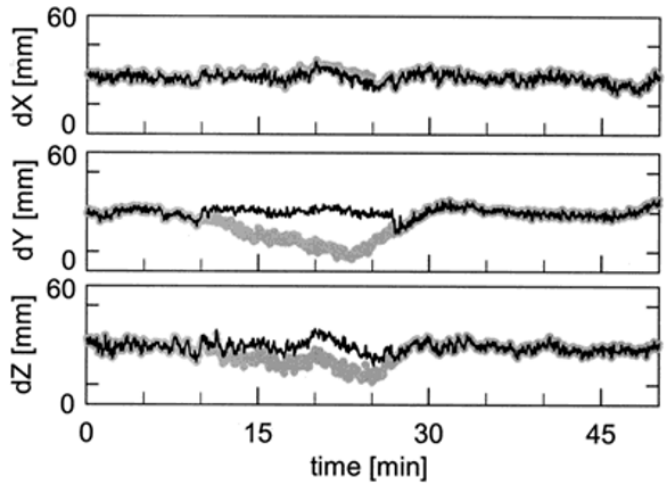

Fig. 11. Time series of coordinates after epoch-to-epoch processing with SIGMA- $\epsilon$ (grey dots) and SIGMA- $\epsilon$ plus Danish Method (solid).

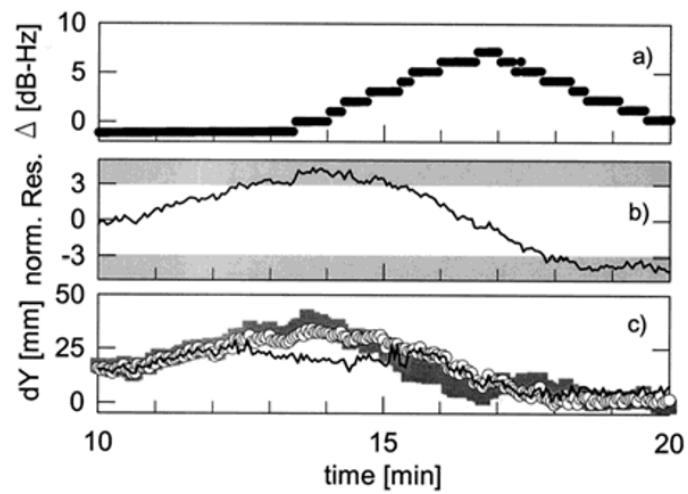

Fig. 12. $\mathrm{C} / \mathrm{N} 0$ attenuation $\Delta$ of PRN29 at rover site (a), normalized residuals of PRN15-29 indicating multipath (b). Deviations dY of epoch-to-epoch dY component from mean of $100 \mathrm{~min}$. (c): SIGMA- $\epsilon$ (circles), SIGMA- $\Delta$ (grey, squares), SIGMA- $\epsilon$ plus Danish Method (solid). $3 \mathrm{~s}$ sampling. 
bias shown by the normalized residuals directly maps into the $\mathrm{dY}$ component of the baseline. This is also indicated by a time series of the epoch-to-epoch baseline solutions (Fig. 12, bottom).

The Danish Method helps in reducing the bias in the result, if the normalized residuals are sufficiently large (depends on good geometry). As can be seen from the dY-plot in Fig. 12, using the SIGMA- $\Delta$ model deteriorates the results in this case. Our investigations show that more than one satellite has significant $\Delta$ values during the period shown, and weighting based on $\Delta$ weakens the geometry. We see also that the Danish Method helps in identifying outliers but cannot remove small systematic errors.

\section{Conclusion}

We have again shown in this paper that the SIGMA- $\epsilon$ weight model based on the $\mathrm{C} / \mathrm{N} 0$ measurements is a good estimate of the actual phase measurement noise. Furthermore, the deviations $\Delta$ of the $\mathrm{C} / \mathrm{N} 0$ values from a template function are valuable indicators for signal distortions due to multipath and diffraction.

With an example dataset for diffraction and one for multipath the limitations of the $\mathrm{C} / \mathrm{N} 0$ based weighting have been demonstrated in this paper. Multipath causes a time lag between $\mathrm{C} / \mathrm{N} 0$ and phase error time series which cannot be easily modelled and thus restricts the proper use of the SIGMA$\Delta$ model. The model performance is better for diffraction effects, but still the $\mathrm{C} / \mathrm{N} 0$ attenuation is no direct measure for the actual phase error. So, the $\mathrm{C} / \mathrm{N} 0$ is not a sufficient tool to correctly reduce biases by weighting observations.

In order to take the information of the residuals into account, we have extended the SIGMA- $\epsilon$ model by the Danish Method. It has been shown that this method of robust estimation helps in the identification and removal of biases. The success of the method depends mainly on good redundancy and on the evaluation of the non-sparse covariance matrix of the residuals. Therefore, the tradeoff between processing all epochs simultaneously (higher redundancy) and process- ing on an epoch-to-epoch basis (small $\mathbf{Q}_{\hat{e}} \hat{e}$ matrix) has to be considered. Moreover, robust estimation is not able to identify small systematic errors but rather effectively eliminates outliers.

The robust estimation procedure investigated in this paper can be used to remove outliers in the phase data. It supports an adaptive weight model, that uses the parameters $\mathrm{C} / \mathrm{N} 0, \Delta$ and normalized residuals along with information about the geometry, redundancy and behaviour of the time evolution of these parameters to determine the most appropriate weights. This generally applicable model is currently developed by the authors. It will allow to further reduce the influence of biased observations on the results for kinematic or short time static position determination with GPS.

\section{References}

Allnutt, J. E., Satellite-to-Ground Radiowave Propagation, 421 pp., Peter Peregrinus Ltd., London, UK, 1989.

Axelrad, P., C. Comp, and P. MacDoran, Use of Signal-To-Noise Ratio for Multipath Error Correction in GPS Differential Phase Measurements: Methodology and Experimental Results, Proc. ION GPS-94, Salt Lake City, Utah, 655-666, 1994.

Baarda, W., A Testing Procedure for Use in Geodetic Networks, 97 pp., Netherlands Geodetic Commission, New Series Vol. 2/5, 1968.

Brunner, F. K., H. Hartinger, and L. Troyer, GPS signal diffraction modelling: The stochastic SIGMA- $\Delta$ model, J. Geod., 73, 259-267, 1999.

Eissfeller, B., Ein dynamisches Fehlermodell für GPS Autokorrelationsempfänger, Schriftenreihe Studiengang Vermessungswesen, Universität der Bundeswehr, München, Heft 55, 1997.

Hartinger, H. and F. K. Brunner, Variances of GPS phase observations: The SIGMA- $\epsilon$ model, GPS Solutions, 2/4, 35-43, 1999.

Jørgensen, P. C., K. Kubik, P. Frederiksen, and W. Weng, Ah, robust estimation!, Aust. J. Geod. Photo. Surv., 42, June, 19-32, 1985.

Krarup, T., J. Juhl, and K. Kubik, Götterdämmerung over least squares adjustment, Proc. 14th Congress of the International Society of Photogrammetry, Hamburg, Vol. B3, 369-378, 1980.

Langley, R., GPS receiver system noise, GPS World, June, 40-45, 1997.

$\mathrm{Xu}, \mathrm{P}$., Consequences of constant parameters and confidence intervals of robust estimation, Boll. Geod. Sc. Affini, 52, 231-249, 1993.

A. Wieser (e-mail: wieser@ivm.tu-graz.ac.at) and F. K. Brunner 